\title{
Misconduct meeting fails to draw sceptics
}

Washington. A conference on scientific misconduct, which was to have included addresses from Donna Shalala, the health secretary, and many leading authorities on misconduct, has been cancelled after only 18 people registered for it.

The meeting, on "The conduct of science: Keeping the faith", was to have been held at the beginning of May at Keystone, Colorado, where the non-profit Keystone Symposia holds its popular and highly regarded meetings on the progress of specific scientific disciplines or subdisciplines.

"We've never [previously] cancelled a meeting in our 22-year history," says Jim Bennett, director of Keystone Symposia and a professor of pharmacology at Michigan State University. The meeting had been publicized through 103,000 standard Keystone leaflets, an advertisement in The Scientist, and detailed programmes sent to the 6,000 subscribers to a newsletter put out by the Department of Health and Human Services' Office of Research Integrity.

Bennett says he did not expect scientists to flock to a meeting whose costs would not have been covered by their grants. But he had hoped that scientific misconduct officers from some of the 800 research universities in the United States, as well as administrators from science funding agencies, would pay the $\$ 175$ fee, enabling Keystone to pay the expenses of the 20 speakers.

The meeting's programme had focused on a report on scientific conduct sent to Shalala and the US Congress last autumn by a commission chaired by Kenneth Ryan of the Brigham \& Women's Hospital, Boston. Critics of the report say that hostility to its findings may have caused the meeting to fail.

"If the community felt that Ryan was

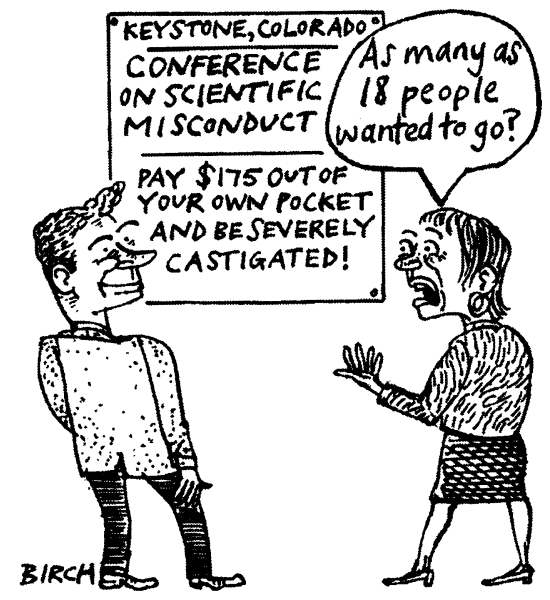

going to be implemented, there would probably have been much more interest," says Sam Silverstein of Columbia University, New York, who was due to speak. He says that what happened "may reflect the status of the Ryan report, which has been disavowed by most interested parties".

Ryan, who would have opened the meeting, was not available for comment. His report has been attacked for proposing a broad definition of sanctionable misconduct, and for embracing topics such as animal abuse and the mishandling of radionuclides, rather than confining itself to what are widely viewed as the 'three deadly sins' of fabrication, plagiarism and falsification.

But Michael Zigmond, a neuroscientist at the University of Pittsburgh who would have addressed the forum on instilling good conduct through education, says that he will miss the chance for the two sides of the debate to confront each other. "If they had asked me, I would have said that very few people would come," says Zigmond. "Most people don't feel that they are experts, and believe that it is not going to effect their own science. But this does not mean that scientists don't care about ethics."

Colin Macilwain
If you've ever developed a purification strategy by yourself, you know there is a lot to consider. Creating media screening schemes; designing buffer preparation routines; selecting which columns to use - even listing the tasks takes careful consideration. But now there's a better way of working. screening. You'll save time as the system automatically recommends the best columns for your runs. You get fast $\mathrm{pH}$ screening as it automatically prepares your buffers from stock solutions. The moment you turn ÄKTAexplorer on, you're presented with a direct path to full-scale purification.

That path is UNICORN ${ }^{\circledR}$ - ÄKTAexplorer's control software. Your scale-up is simplified as the system's software can transfer and implement your methods on purification systems at all scales.

Of course, these are just a few of ÄKTAexplorer's features. So call us at I (800) 5263593 from North America, +81 (0)3 34926949 from Japan or $+46(0) 18 \quad 1650 \quad$ । I from the rest of the world (or meet us on the Internet at http://www.biotech.pharmacia.se/akta.htm). scouting and media

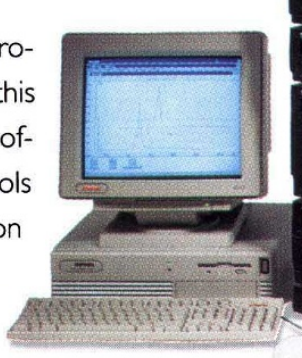

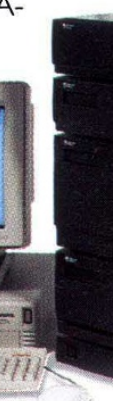

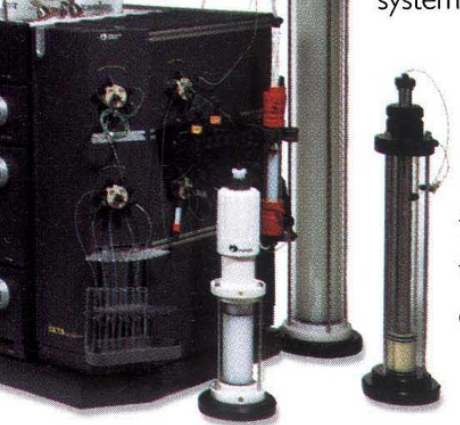

